\title{
COMPARISON BETWEEN VISUAL AND TACTILE SIGNAL DETECTION TASKS APPLIED TO THE SAFETY ASSESSMENT OF IN-VEHICLE INFORMATION SYSTEMS
}

\author{
Johan Engström, Nina Åberg, Emma Johansson \\ Department for Human Systems Integration \\ Volvo Technology Corporation, Göteborg, Sweden \\ E-mail: johan.a.engström@volvo.com \\ Jakob Hammarbäck \\ Uppsala, Sweden
}

\begin{abstract}
Summary: The Peripheral Detection Task (PDT) is a widely applied method for safety assessment of in-vehicle information systems (IVIS). In this study, the PDT was compared to a Tactile Detection Task (TDT) where the visual stimulus used for the PDT was replaced by tactile vibrators, placed on the wrists. The sensitivity of the two methods to different cognitive and visual secondary tasks was investigated in different real-world driving conditions. The results showed that both methods were sensitive to visual and cognitive secondary task load in a range of different driving environments. The sensitivity was generally stronger for the TDT than the PDT. It was concluded that the TDT could be a viable alternative to the PDT for IVIS assessment.
\end{abstract}

\section{INTRODUCTION}

There is a growing concern over the potentially hazardous effects that in-vehicle information systems (IVIS) may have on road safety, in particular with respect to excessive cognitive and/or visual distraction. In order to ensure safe IVIS design, there is a need for valid, reliable and costefficient methods for quantifying these effects. One such method that has become increasingly popular is the Peripheral Detection Task (PDT), first described in van Winsum, Martens and Herland (1999). The PDT measures the ability to detect visual stimuli presented in the peripheral field of view with a certain temporal and spatial uncertainty. In field trials, the stimuli are typically generated by LEDs (Light Emitting Diods) placed on the dashboard and reflected in the windshield. An alternative approach, used for example by Schindhelm et al. (2003), is to use a single head-mounted LED placed in the driver's peripheral view. Responses are typically given by means of a button attached to the index finger. Detection performance is measured in terms of response time and hit rate.

PDT has proven sensitive to both visually and cognitively loading secondary tasks (Olsson and Burns, 2000) as well as differences in driving task demand (van Winsum, et al., 1999). The method has been applied to IVIS evaluation in several studies, including the comparison of GPSbased route guidance to memory-based navigation (Harms and Patten, 2003), the evaluation of speech- and tactile messages in the IN-ARTE driving support system (van Winsum et al., 1999), the comparison between speech-based and manual dialogues for navigation systems (Israelsson and Karlsson, 2003) and the assessment of the COMUNICAR workload manager (Schindhelm et al., 2003). It was also the primary method used in the major Swedish investigation of the roadsafety effects of mobile phone use while driving (Patten, Kircher, Östlund and Nilsson, 2003). 
However, despite its wide application, the PDT method still has a number of unresolved issues associated with it. First, the conspicuity of the stimuli varies strongly with lighting conditions. A second problem concerns the relation between the two performance measures (hit rate and response time). In demanding situations, drivers may shift strategy and respond fast and accurately only to a subset of the stimuli. This would lead to a reduced hit rate but unaffected response times, which makes the data difficult to compare to "normal" data (with unaffected hit rate and increased response times). Thus a single response measure would make the data easier to interpret. Finally, the PDT could be expected to affect visual scanning, which makes simultaneous recording of eye-movement data problematic. Thus, if a study requires collection of both PDT and eye movement data, these need to be collected separately, which greatly increases cost and labour.

A possible alternative to the PDT would be a signal detection task where stimuli are given in a different sensory modality. This is based on the hypothesis that the reduced detection performance measured by the PDT is the result of a general interference in human information processing rather than a modality-specific perceptual interference. For visual detection tasks, the latter idea is often referred to as the visual tunneling hypothesis (e.g., Miura, 1986). A number of recent results, from detection task studies (van Winsum et al., 1999; Recarte and Nunes, 2003) as well as brain-imaging experiments (Just et al., 2001) appear to support the general interference hypothesis, at least for secondary tasks-related workload.

The objective of the present study was to investigate a tactile alternative to the PDT, henceforth referred to as the Tactile Detection Task (TDT). The TDT differs from the PDT only with respect to the stimuli, which are presented by means of small vibrators placed on the wrists. Since the TDT is not subject to problems with lighting conditions, it is potentially more reliable and sensitive than the PDT. It is also likely that the hit rate in highly demanding situations could be somewhat increased with the TDT, which would give more reliable response time measures, thus facilitating its use as a single performance measure. The TDT would also yield a "pure" measure of cognitive load not mixed up with the effect of simply looking away. Moreover, since the TDT does not influence eye movements, visual demand measures can be obtained simultaneously. The study should also shed further light on the theoretical issue of general interference versus visual tunneling. If the TDT yields similar results as the PDT, across a range of loading tasks, it would be a strong support for the general interference hypothesis.

\section{METHOD}

\section{Subjects}

Thirteen subjects, 7 men and 6 women, participated in the study. They were all employees at Volvo Technology, but with no specific knowledge or expertise about the subject of the study. Their age ranged from 19 to 53 years (mean 30). The subjects had had their driver's license for 0.5 to 34 years (mean 10) and drove 1000-3000 km per year (mean $2000 \mathrm{~km}$ ). All subjects were right-handed. 


\section{Driving environments}

Three different driving environments were included in the study: motorway, rural road and city driving. The motorway was a standard Swedish motorway outside Göteborg, with a posted speed limit of $110 \mathrm{~km} / \mathrm{h}$ and divided lanes (two in each direction). For the rural road, with one lane in each direction, the speed limit varied between 50,70 and $90 \mathrm{~km} / \mathrm{h}$. Finally, city driving took place in Göteborg city centre. The trials took place during summer in daylight conditions.

\section{Secondary tasks}

The subjects performed three different secondary tasks, two purely cognitive (Question and Counting) and one visual/cognitive (Phone). The Question task was to answer simple yes/no questions. The Counting task was to count down with increments of seven from a given threedigit number. Finally, in the Phone task, the subjects were asked to dial a 12-digit number, using a hand-held mobile phone. The number was given by a post-it note placed on the centre console on the dashboard. In addition, a baseline condition (with no secondary task) was included.

\section{The signal detection tasks}

The set-up for the PDT followed the general specifications described in van Winsum et al. (1999) and Olsson and Burns (2001). However, the positioning of the LEDs was somewhat different compared to previous studies. In order to increase the visual conspicuity of the LEDs, they were placed in a position where they could be directly perceived by the driver (i.e., not reflected in the windscreen). The tactile stimuli were given by means of two small electrical vibrators, attached to the left and right wrists. The stimuli were varied randomly between the two vibrators.

For both the PDT and the TDT, responses were given by means of a small button attached to the left index finger. Detection performance was measured in terms of response time and hit rate. Hits were defined as stimuli responded to within 2 seconds from stimulus onset, with the exception of unrealistically fast responses ( $<0.2$ seconds). Hit rate was defined as the number of hits divided by the total number of stimuli during a task or baseline condition. Response time was defined as the average response time of the valid responses during a secondary task or a baseline section.

\section{Design}

The independent factors in the experiment were Secondary Task, Driving Environment and Stimulus Modality. In general, a $4 \times 3 \times 2$ within-group design was employed, with four Secondary Task conditions (Baseline - no task, Question, Counting and Phone), three Driving Environments (Motorway, Rural road and City) and two Stimulus Modalities (Visual, Tactile). The order of the different conditions was counterbalanced between subjects to control for learning effects. The dependent variables were hit rate and response time. 


\section{Procedure}

Before the trial, the subjects completed a form with data on age and driving experience, and signed a consent form. The drivers also practiced the secondary tasks and the signal detection tasks. During, the trial, a test leader was positioned in the back seat and gave instructions about the route and when to perform the secondary tasks. The total test time was 1-1.5 hours.

\section{RESULTS}

In a first analysis, separate univariate ANOVAs were carried out for each of the two methods (PDT and TDT), with Task and Driving Environment as factors. The results are illustrated in Figure 1 and further described below.
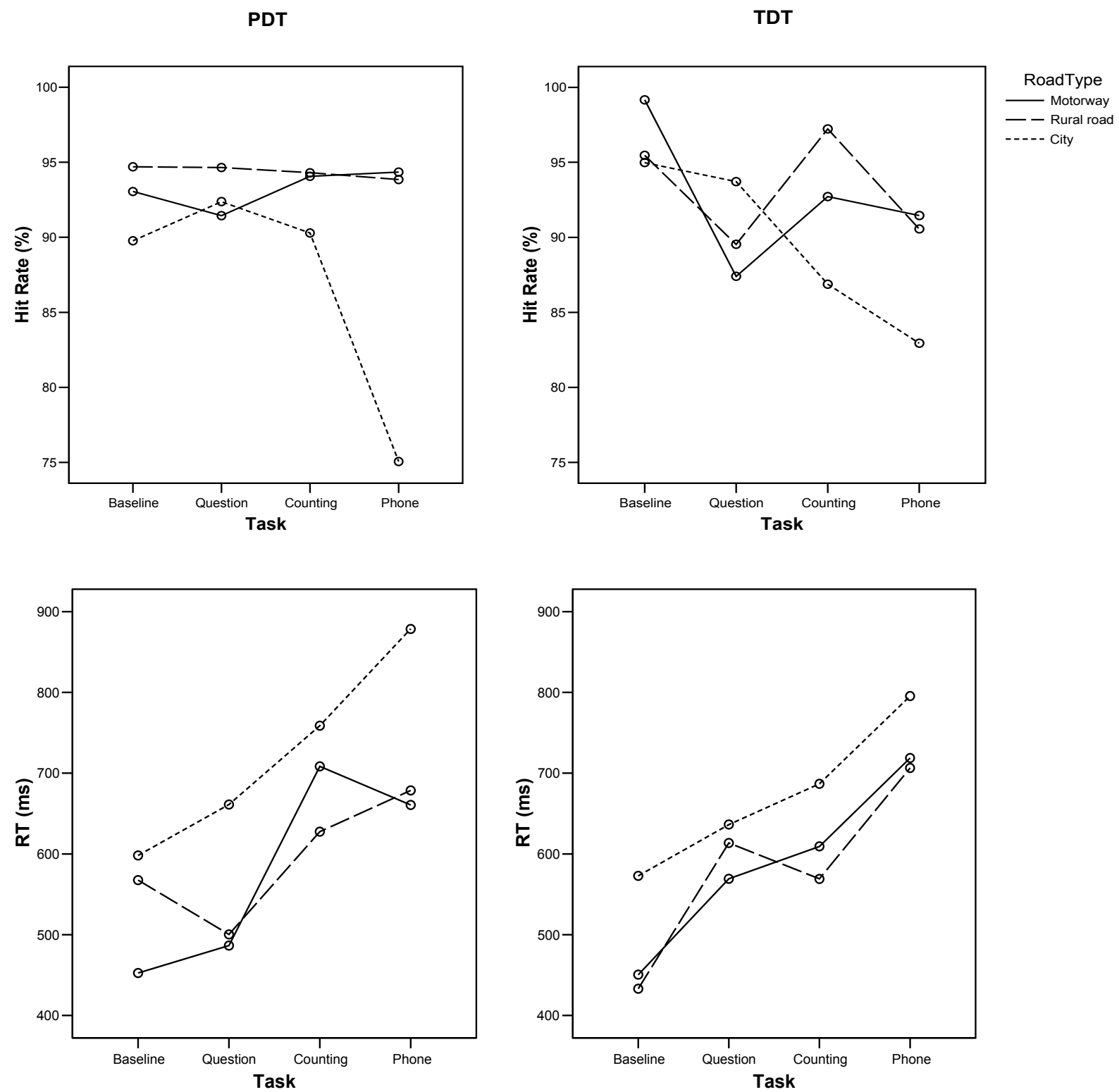

Figure 1. Hit rates (top) and response times (bottom) for the PDT (left) and the TDT (right) 


\section{PDT (visual stimuli)}

For hit rate, no significant effects were found for Secondary Task. However, a small main effect was found for Driving Environment $(F(2,22)=3.85, p<.05)$. Sidak post hoc testing revealed that the only significant difference was that between City and Rural Road. In general, the hit rate was high and exceeded $88 \%$ in all conditions except Phone-City, where it dropped to $75 \%$.

For response time, significant main effects were found for both Secondary Task $(F(3,33)=12.6$, $p<.001)$ and Driving Environment $(F(2,22)=6.0, p<.01)$. For Driving Environment, post hoctesting showed that response times in the City were significantly longer than those in the Motorway and the Rural Road conditions. For Secondary Task, significant differences to baseline were found for Counting and Phone, but not the Question task. The results are illustrated in Figure 1.

\section{TDT (tactile stimuli)}

For the TDT, no effects were found for hit rate. Like for the visual modality, the hit rates were generally high: $>86 \%$ in all conditions but Phone-City. In this case, however, the drop was not as drastic as for the PDT.

For response time, main effects were found for both Secondary Task $(F(3,33)=22,3, p<.001)$ and Driving Environment $(F(2,22)=7.5, p<.01)$. Sidak post-hoc analysis for Driving Environment showed that the City condition was significantly different from Motorway and Rural Road. For Secondary Task, all differences between baseline and the three tasks were significant $(p<.001)$. The results are illustrated, and compared to the PDT results, in Figure 1.

\section{Comparison between PDT and TDT}

In order to further compare the two methods, a univariate ANOVA was performed, with Stimulus Modality as an additional independent factor. No effect of Stimulus Modality was found, neither for hit rate nor response time. However, a small but significant interaction effect was found for Stimulus Modality and Secondary Task for response time $(F(3,193)=2.94$, $p<0.05)$. The overall mean response time was $632 \mathrm{~ms}$ for the PDT and $613 \mathrm{~ms}$ for the TDT. The corresponding hit rates were $91.5 \%$ and $91.8 \%$ respectively.

The PDT and TDT response times were further compared in terms of the estimated effect sizes for the three secondary tasks (i.e., the standardised differences between each task and baseline). The results are illustrated in Figure 2. It can be observed that the effect is substantially larger for the TDT for the Question and Phone task. For the Question task, the PDT was not sensitive at all. 


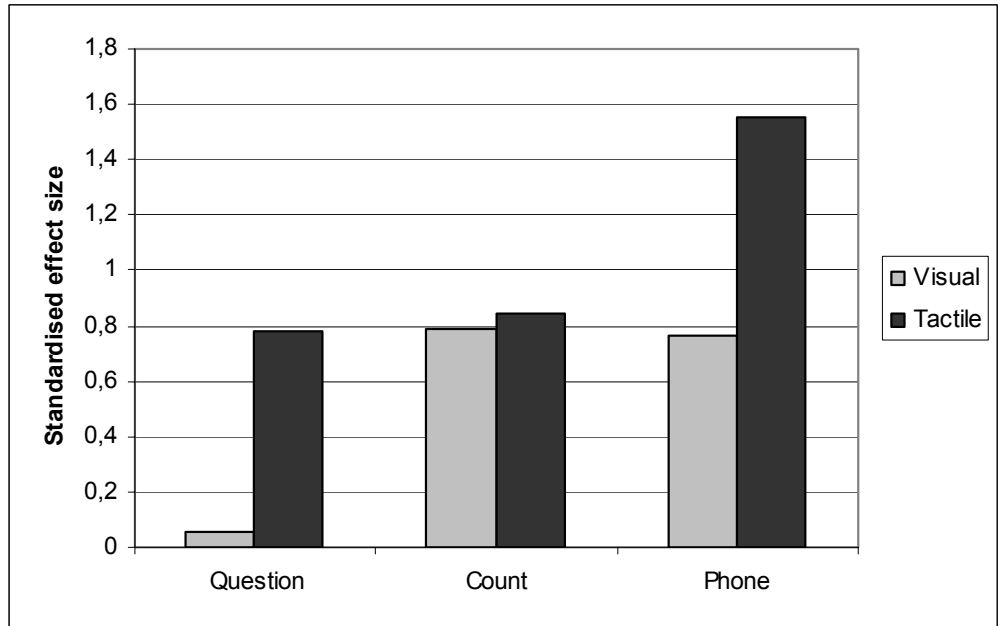

\section{Figure 2. Comparison of effect sizes for the visual (PDT) and the tactile (TDT) modality for response time}

\section{DISCUSSION}

The results show that response times generally increased as a function of secondary task load as well as environment complexity, both for the PDT and the TDT. However, only the TDT was sensitive to the Question task and the TDT was also substantially more sensitive to the Phone task than the PDT. The latter result is especially surprising, given that the phone task involved a visual component which might be expected to interfere more with the visual PDT than the tactile TDT. Clear examination of the data showed that the larger effect size for TDT for the phone task (Figure 2) was mainly due to larger between-subject variance in the PDT data, possibly partly induced by changing light conditions.

The response times were also significantly longer in city driving, compared to the motorway and the rural road. This strong sensitivity (of both detection tasks) to environment complexity is in line with existing results (e.g., van Winsum et al., 1999), and stresses the need to keep the environmental complexity constant in the evaluation scenarios. However, it is also clear that both the PDT and the TDT worked well (i.e., were sensitive to secondary task load) also in the most demanding driving environment (City).

For hit rate, weak effects or no effects at all were found, which indicates that response time is the more appropriate performance measure, while hit rate seems better suited as a quality measure. Although the overall hit rates were very similar between PDT and TDT, the former was somewhat less robust in demanding conditions, as indicated by the radical drop to $75 \%$ in the Phone-City condition.

Barring the differences just discussed, the results for PDT and TDT were generally similar. Both methods were sensitive to different types of secondary tasks and environment complexity and the overall mean response times and hit rates were remarkably similar. This suggests that the observed reduction in signal detection performance in loading conditions is largely independent of the perceptual modality of the stimulus. This strongly supports the general interference hypothesis. The common resource pool where this interference takes place is probably associated 
with higher cognitive functions such as selective attention. Some hints of the nature of this common resource pool, based on neurophysiological data, are offered by Just et al. (2001).

From a practical perspective, the present results clearly show that the TDT can be regarded as a viable alternative to the PDT. A key advantage of the TDT is that it provides a "pure" quantification of the response delay induced by cognitive load, not affected by the fact that the driver is simply looking away from the target. As such, it is a good complement to eye movement-based measures of visual demand. Moreover, by contrast to the PDT, TDT data do not affect eye movements and the two types of data can thus be collected simultaneously. However, it should be pointed out that the PDT still has a certain advantage with respect to face validity. The ability to detect visual stimuli in the forward view has a direct and obvious relation to safety, and some of this face validity is lost with the TDT. However, on the assumption of the general interference hypothesis, the TDT could be regarded as a valid surrogate for the PDT. However, more empirical and theoretical work is needed to firmly establish the TDT as a valid IVIS safety evaluation method.

\section{REFERENCES}

Harms, L., a Patten, C. (2003). Peripheral detection as a measure of driver distraction. A study of memory-based versus system-based navigation in a built-up area. Transportation Research, Part F 6: 23-36.

Israelsson, E., \& Karlsson, N. (2003). How does a speech user interface affect the driving task? In, P. McCabe (Ed.). Contemporary Ergonomics 2003. London: Taylor and Francis.

Just, M. A., Carpenter, P.A., Keller, T.A., Emery, L., Zajac, H. \& Thulborn, K. R. (2001). Interdependence of non-overlapping cortical systems in dual cognitive tasks. Neuroimage, 14: 417-426.

Miura, T. (1986). Coping with Situational Demand: A Study of Eye Movements and Peripheral Vision Performance. In, Gale et al. (Eds.). Vision in Vehicles, pp. 205-217. Elsevier Science Publishers.

Olsson, S., \& Burns, P. (2000). Measuring distraction with a peripheral detection task. On-line paper. NHTSA Internet Distraction Forum. Available at www.nrd.nhtsa.dot..gov/ departments/nrd-13/driver-distraction/welcome.htm

Patten, C., Kircher, A., Östlund, J., \& Nilsson, L. (2003). Using mobile telephones: cognitive workload and attention resource allocation. Accident Analysis and Prevention, 36(3): 341350 .

Recarte, M., \& Nunes, L. (2003). Mental Workload While Driving: Effects on Visual Search, Discrimination, and Decision Making. Journal of Experimental Psychology: Applied. Vol. 9, No. 2, 119-137.

Schindhelm, R., Gelau, C., Montanari, R., Morreale, D., Deregibus, E., Hoedemaeker, M., de Ridder, S., \& Piamonte, P. (2003). Human factor tests on car demonstrator. Deliverable no D6.4. Final draft. COMUNICAR Programme IST KA1. Testing demonstration and pilot evaluation Work package WP6. Report nr: 2003 P 134. 
PROCEEDINGS of the Third International Driving Symposium on Human Factors in Driver Assessment, Training and Vehicle Design

van Winsum, W., Martens, M., \& Herland, L. (1999). The effect of speech versus tactile driver support messages on workload, driver behaviour and user acceptance. TNO-report TM-99C043. Soesterberg, Netherlands. 